\title{
Presentación, manejo y evolución de pacientes con herida por proyectil de arma de fuego en el abdomen. Una década de violencia en México
}

\author{
Presentation, management and evolution of patients with abdominal gunshot wound. A \\ decade of violence in Mexico
}

Daniel R. Cantú-Alejo ${ }^{1}$, Francisco Reyna-Sepúlveda" ${ }^{*}$, Saúl García-Hernández ${ }^{1}$, Jessica Sinsel-Ayala ${ }_{-}^{1}$ Marco Hernández-Guedea ${ }^{1,2}$, Edelmiro Pérez Rodríguez ${ }^{1}$ y Gerardo Muñoz-Maldonado ${ }^{1}$

${ }^{1}$ Departamento de Cirugía General; 2Departamento de Urgencias. Hospital Universitario "Dr. José Eleuterio González", Universidad Autónomăa de Nuevo León, Monterrey, Nuevo León, México

\section{Resumen}

Antecedentes: Las lesiones traumáticas representan el 9.6\% de la carga global de la enfermedad y son la tercera causaक̣nás común de muerte en adultos y adolescentes. Entre los pacientes ingresados en el hospital con lesiones traumáticas, el-fraumatismo penetrante abdominal sigue siendo una importante causa de morbilidad y mortalidad. Objetivo: Analizar la prêsentación, el manejo y la evolución de los pacientes con herida por proyectil de arma de fuego en el abdomen. Método: Estudio retrospectivo, descriptivo y observacional. Se revisaron todos los pacientes con diagnóstico de herida por proyectil de ằrma de fuego en el abdomen en el departamento de estadística del Hospital Universitario Dr. José E. González durante los âños 2010-2015. Resultados: Del total, 21 (9.1\%) pacientes eran del sexo femenino y 229 (90.9\%) del sexo masculino. Se ingresaron con un promedio de $1.22 \pm 0.51$ lesiones, con una puntuación en la Escala de coma de Glasgow de $13.59 \pm 2.5$, saturación de oxígeno de $96.2 \pm 2.09$, frecuencia cardiaca de $94.4 \pm 20$, frecuencia respiratoria de $22 \pm 6$ y grado de chóque de $1.5 \pm 0.80$ Conclusión: Los signos vitales y las características específicas de la lesión son esenciales y útiles para la predicción de la mortalidad por traumatismo abdominal por proyectil de arma de fuego, además de la cuantificación de heridas y lesiones orgánicas asociadas, siendo evaluadas estas tanto en un todo como por separado.

Palabras clave: Abdomen. Estancia hospitalaria. Herida por proyectil de arma de fuego. Mortalidad. Traumatismo.

\begin{abstract}
Background: Traumatic injuries represent $9.6 \%$ of the global surface of the disease and represent the third most common cause of death in adults and adolescents. Patients were admitted to the hospital presenting traumatic injuries, penetrating abdominal traumatism remains a major cause of morbidity and mortality. Objective: To analyze the presentation, handlingand development of patient's gunshot wound. Method: Retrospective, descriptive, observational study. It was reviewed from 2010 2015 in the statistics department of the University Hospital Dr. José E. González all patients diagnosed with firearm projectile injury in abdomen. Results: Were admitted above an average of $1.22 \pm 0.51$ lesions, on a Glasgow coma scale of $13.59 \pm$ 2.5 , oxygen saturation of $96.2 \pm 2.09$, heart rate of $94.4 \pm 20$, respiratory rate of $22 \pm 6$ and shock degree of $1.5 \pm 0.80$.

\section{Correspondencia:}

*Francisco Reyna-Sepúlveda

Francisco I. Madero Pte. y Av. Gonzalitos, s/n

Col. Mitras

Fecha de recepción: 24-11-2019

C.P. 64460 , Monterrey, N.L., México

E-mail: Francisco.reynas@uanl.mx

0009-7411/@ 2020 Academia Mexicana de Cirugía. Publicado por Permanyer. Este es un artículo open access bajo la licencia CC BY-NC-ND
\end{abstract}


Conclusion: Vital signs and specific characteristics of the lesion are essential and useful for the prediction of mortality due to abdominal traumatism by firearm projectile, in addition to the quantification of wounds and associated organic lesions, these are being evaluated both as a whole and separately.

Key words: Abdomen. Hospital stay. Firearm projectile injury. Mortality. Trauma.

\section{Introducción}

Las lesiones traumáticas representan el $9.6 \%$ de la carga global de la enfermedad y son la tercera causa más común de muerte en adultos y adolescentes ${ }^{1,2}$. Entre los pacientes ingresados en el hospital con lesiones traumáticas, el trauma abdominal (TA) penetrante por herida por proyectil de arma de fuego (HPPAF) sigue siendo una importante causa de morbilidad y mortalidad ${ }^{3-5}$. En todo el mundo, las lesiones por arma de fuego homicida se producen a una tasa de $0.2-4.8$ por 100.000 habitantes $^{6}$, con una tasa de mortalidad cercana al $88 \% 5$.

Las mejoras en los sistemas de trauma en los países de altos ingresos, como los Estados Unidos de Norteamérica, han resultado en una mejora significativa en la supervivencia de los pacientes con TA penetrante (incluyendo heridas de bala) ${ }^{7-9}$. Para los países de ingresos bajos y medianos, donde la carga de lesiones por arma de fuego es enorme ${ }^{10-12}$, sigue siendo necesario establecer y conocer su presentación y su actual evolución, datos que no se conocen en México.

En México, las lesiones por traumatismo han sido relevantes dentro de la última década, con un notable incremento anual. Se vuelven especialmente relevantes en adultos jóvenes, ya que representaron el 50\% de la mortalidad entre los 15 y los 34 años. En el año 2016 se registraron 72,821 muertes violentas. Los órganos mayormente afectados por TA por HPPAF son el intestino delgado (50\%), el intestino grueso $(40 \%)$ y el hígado $(30 \%)^{13}$.

En el presente estudio nos planteamos dos hipótesis:

- La presencia de choque y el número de lesiones orgánicas abdominales representan variables de interés para la estratificación del TA y la HPPAF.

- Existen variables acompañantes del TA por HPPAF que modifican la mortalidad y el tiempo de estancia hospitalaria.

El objetivo primario fue proporcionar una comparación directa de la mortalidad con los diferentes escenarios de los pacientes, y así determinar las variables de peor pronóstico y alta mortalidad.
Los objetivos secundarios fueron postular variables para optimizar los métodos de estratificación en-los países en vías de desarrollo y realizar un análisišs de riesgo para estancia hospitalaria prolongada $\geq 14$ días).

\section{Método}

Estudio retrospectivo, descriptivo y observacional. Se revisaron 230 expedientes clínicos de pacientes admitidos en el departamento de cirugía del Hospital Universitario José Eleuterio González, del año 2011 al 2015, que presentasen TA por HPPAF abdominal. El tamaño de muestra se determinó por el máximō de casos viables para el estudio encontrados en el loperiodo de tiempo señalado $(n=230)$.

El presente estudio fue llevado a cabo bajo la torización del comité de investigación de la institución concerniente. La información obtenida fue recoleẹtada en una base de datos electrónica para su posterior uso en el análisis estadístico. La información privāada de los pacientes fue revisada solo por los autores y coautores, y como protocolo de privacidad se eliminarán los archivos digitales en un periodo de 5 años.

Esta investigación se sometió a revisión por el.p̈ersonal del departamento de cirugía del Hospital Üniversitario José Eleuterio González.

- Criterios de inclusión: las operaciones concernientes a este estudio serán realizadas deñitro del Hospital Universitario José Eleuterio González; paciente que haya sufrido TA por HPPAE.

- Criterios de exclusión: el expediente médico que revisar no contiene la información concerniēnte para este estudio; el expediente clínico presenta irregularidades en su contenido.

Las variables consideradas fueron obtenidas tras la revisión de los expedientes clínicos resguardados el departamento de archivo del Hospital Universitario José Eleuterio González. Dicha revisión se realizó por cada uno de los diferentes formatos que conforman el expediente. Los expedientes clínicos fueron revisados exclusivamente por los autores de esta investigación.

- Clínica: órgano lesionado, tipo de lesión, si fueúnica o múltiple, y cuadrante de la lesión o lesiones. 
- Exploración física: Escala de coma de Glasgow, signos vitales incluyendo frecuencia cardíaca, frecuencia respiratoria, presión arterial, temperatura y saturación de oxígeno.

- Cirugía: tipo de lesión, tiempo quirúrgico, paquetes globulares, si requirió terapia intensiva.

Los datos se analizaron usando el programa informático Numbers ${ }^{\circledR}$ v3.6.1 (Apple Inc.) y el análisis estadístico con el programa IBM SPSS Statistics v20.0 (SPSS, Inc., Armonk, NY, EE.UU.), tablas de contingencia y prueba de ji al cuadrado².

\section{Resultados}

Se obtuvieron 237 casos, pero siete no contaban con expediente clínico completo, pudiendo aumentar el factor de confusión, y por ello se revisaron un total de 230 casos, de los cuales 22 (9.6\%) eran del sexo femenino y $208(90.4 \%)$ eran del sexo masculino (Fig. 1), del año 2011 al 2015, con TA por HPPAF, con la distribución reflejada en la Tabla 1.

Los datos sociodemográficos se mencionan en la tabla 2. Además, se realizó un análisis para lesiones orgánicas y si incrementan o no el riesgo de muerte al estar presentes, estado de choque y cantidad de lesiones orgánicas y de heridas penetrantes.

En cuanto a las variables clínicas, se ingresaron con un promedio de $1.22 \pm 0.51$ lesiones, con una puntuación en la Escala de coma de Glasgow de $13.59 \pm 2.5$, saturación de oxígeno de $96.2 \pm 2.09$, frecuencia cardiaca de $94.4 \pm 20$ latidos por minuto, frecuencia respiratoria de $22 \pm 6$ respiraciones por minuto, grado de choque de $1.5 \pm 0.80$, TRISS (Trauma and Injury Severity Score) promedio de 19 y RTS (Revised Trauma Score) de 6.4. Se identificaron 64 pacientes con irritación peritoneal.

El tiempo de internamiento promedio fue de 14.28 \pm 19.82 días. Un total de 41 pacientes pasaron a terapia intensiva, donde permanecieron $1.29 \pm 3.2$ días. Se realizaron un promedio de $3.4 \pm 4.1$ transfusiones. Se identificaron 28 pacientes sin lesión. Del total de 230 pacientes, solo 147 presentaron lesiones únicamente en el abdomen, 69 en el abdomen y el tórax, 11 en el abdomen y las extremidades, y 4 en el abdomen y el cuello. La mortalidad reportada fue de 27 (11.7\%). Los factores de mortalidad asociada a estancia hospitalaria prolongada se observan en la tabla 3 , incluyendo signos vitales, heridas y cuadrante abdominal afectado.

El factor de confusión se encuentra presente por la falta de diferenciación de variables entre tiempos

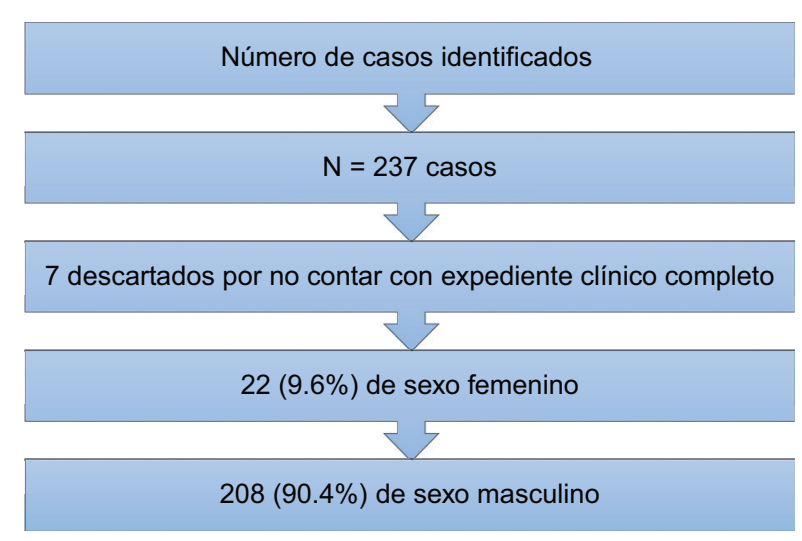

Figura 1. Número de casos identificados tras el análisis de la bašse de datos electrónica.

Tabla 1. Pacientes asistidos por herida abdominal por proyectil de arma de fuego por año $(n=230)$

\begin{tabular}{|c|c|}
\hline Año & Pacientes \\
\hline 2011 & $56^{\circ}$ \\
\hline 2012 & 63 \\
\hline 2013 & $54^{\circ}$ \\
\hline 2014 & $22_{n}$ \\
\hline 2015 & $28 \frac{3}{0}$ \\
\hline Total & 230 \\
\hline & $\begin{array}{l}\overline{0} \\
\overline{0} \\
\frac{0}{0} \\
\end{array}$ \\
\hline
\end{tabular}

preoperatorios, transoperatorios y posoperatorios. Se reduce el factor de confusión al cuantificar todasilas variables de interés.

\section{Discusión}

Esta investigación tuvo limitaciones en cuanto a obtención de variables preoperatorias, transoperatorias y posoperatorias, además de por la interpretación de los expedientes clínicos dada a los diferentes criterios quirúrgicos utilizados para la toma de decisisiones. Actualmente se dispone de una gran cantida de medidas de gravedad de la lesión para cuantificar y estratificar el riesgo de lesiones abdominales pềnetrantes ${ }^{14-18}$. La presencia de choque y el número de órganos abdominales dañados han demostrado ser marcadores de gravedad fiables en los pacientes con TA por HPPAF ${ }^{19}$.

Del mismo modo, los retrasos en las intervenciones quirúrgicas y una alta puntuación de gravedad de la lesión (PGL) también se han relacionado con altas 
Tabla 2. Datos sociodemográficos y mortalidad asociada en pacientes con herida abdominal por proyectil de arma de fuego $(n=230)$

\begin{tabular}{|c|c|c|c|c|c|}
\hline & General $(n=230)$ & Análisis estadístico & Mortalidad $(n=28)$ & No mortalidad ( $\mathrm{n}$ & $=202$ \\
\hline Mortalidad & $28(12 \%)$ & & $28(100 \%)$ & $202(100 \%)$ & $\stackrel{\grave{2}}{2}$ \\
\hline \multicolumn{6}{|l|}{ Número de heridas } \\
\hline 1 & $131(56 \%)$ & & $5(17 \%)$ & $127(62 \%)$ & $\frac{\xi}{4}$ \\
\hline$\geq 2$ & $98(42 \%)$ & & $23(82 \%)$ & $75(37 \%)$ & $\frac{1}{2}$ \\
\hline \multicolumn{6}{|l|}{ Cuadrante abdominal afectado } \\
\hline Superior derecho & $80(34 \%)$ & NS & $9(32 \%)$ & $77(38 \%)$ & . \\
\hline Superior izquierdo & $60(26 \%)$ & NS & $7(25 \%)$ & $50(24 \%)$ & : \\
\hline Inferior derecho & $30(13 \%)$ & NS & $5(17 \%)$ & $30(14 \%)$ &.$\underline{\underline{u}}$ \\
\hline Inferior izquierdo & $60(26 \%)$ & NS & $7(25 \%)$ & $55(27 \%)$ & $\overline{\frac{0}{2}}$ \\
\hline \multicolumn{5}{|l|}{ Grado de choque } & ס \\
\hline 1 & $159(69 \%)$ & & $14(50 \%)$ & $155(76 \%)$ & ฮ) \\
\hline 2 & $59(25 \%)$ & & $10(35 \%)$ & $44(21 \%)$ & $\frac{0}{0}$ \\
\hline$\geq 3$ & $10(4 \%)$ & & $4(14 \%)$ & $4(2 \%)$ & 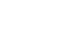 \\
\hline \multicolumn{6}{|l|}{ Sexo } \\
\hline Masculino & $208(90 \%)$ & & $28(100 \%)$ & $181(89 \%)$ & $\underset{\check{C}}{\stackrel{\sigma}{c}}$ \\
\hline Femenino & $22(10 \%)$ & & 0 & $21(10 \%)$ & $\frac{5}{5}$ \\
\hline Edad & $29.5 \pm 11.2$ & NS & $30 \pm 13.5$ & $29.5 \pm 10.9$ & 9. \\
\hline \multicolumn{6}{|l|}{ Días de internamiento } \\
\hline >14 días & $47(20 \%)$ & NS & $10(35 \%)$ & $58(28 \%)$ & $\frac{\overline{0}}{2}$ \\
\hline Terapia intensiva & $50(21 \%)$ & & $22(78 \%)$ & $28(13 \%)$ & 응 \\
\hline \multicolumn{6}{|l|}{ Sitio de lesión } \\
\hline Abdomen & $147(63 \%)$ & & $14(50 \%)$ & $133(65 \%)$ & 4 \\
\hline Abdomen y otro sitio & $82(35 \%)$ & & $14(50 \%)$ & $69(34 \%)$ & 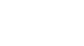 \\
\hline Lesiones asociadas & $77(33 \%)$ & NS & $14(50 \%)$ & $63(31 \%)$ &.$\triangleq$ \\
\hline Glasgow & $14.3 \pm 1.3$ & 0.033 & $13 \pm 1$ & $14 \pm 1$ & "록 \\
\hline \multicolumn{6}{|l|}{ Signos vitales } \\
\hline Temperatura & $36.3 \pm 0.41$ & 0.035 & $36 \pm 0.5$ & $36 \pm 1$ & $\frac{0}{2}$ \\
\hline Saturación < 92 & $14(6 \%)$ & NS & $3(10 \%)$ & $11(5 \%)$ & $-\frac{1}{2}$ \\
\hline Frecuencia cardiaca $>100$ & $80(34.8 \%)$ & NS & $18(64 \%)$ & $62(30 \%)$ & 흔 \\
\hline Presión sistólica & $120 \pm 21$ & 0 & $121 \pm 19$ & $119 \pm 21$ & 을 \\
\hline Presión sistólica < 90 & $28(12.2)$ & NS & $2(7 \%)$ & $26(13 \%)$ & 읃 \\
\hline Frecuencia respiratoria > 20 & $139(60 \%)$ & NS & $21(75 \%)$ & $118(58 \%)$ & \pm \\
\hline Frecuencia respiratoria $<12$ & $6(2 \%)$ & NS & $1(3.5 \%)$ & $5(3 \%)$ & 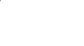 \\
\hline \multicolumn{5}{|l|}{ RTS } & $\frac{\pi}{0}$ \\
\hline $3-10$ & $230(100 \%)$ & & $28(100 \%)$ & $202(100 \%)$ & $\frac{0}{0}$ \\
\hline Irritación peritoneal & $68(29 \%)$ & NS & $12(42 \%)$ & $56(27 \%)$ & 운 \\
\hline Tórax & $69(30 \%)$ & NS & $12(42 \%)$ & $57(28 \%)$ & "- \\
\hline Tráquea & $31(13 \%)$ & NS & $3(10 \%)$ & $28(13 \%)$ & ฮั \\
\hline Esófago & $37(16 \%)$ & NS & $8(28 \%)$ & $29(14 \%)$ & ᄒ̀ \\
\hline Duodeno & $12(5 \%)$ & 0.043 & $8(28 \%)$ & $4(2 \%)$ & $\frac{2}{0}$ \\
\hline Intestino delgado & $72(31 \%)$ & 0 & $12(42 \%)$ & $60(39 \%)$ & $\frac{0}{3}$ \\
\hline Intestino grueso & $99(43 \%)$ & NS & $13(46 \%)$ & $86(42 \%)$ & 凹 \\
\hline Bazo & $34(14 \%)$ & NS & $5(17 \%)$ & $29(14 \%)$ & $\frac{2}{0}$ \\
\hline Hígado & $59(25 \%)$ & NS & $9(32 \%)$ & $50(24 \%)$ & $\stackrel{\vec{x}}{\stackrel{+}{c}}$ \\
\hline Páncreas & $13(5 \%)$ & NS & $5(17 \%)$ & $8(4 \%)$ &.$\frac{\bar{\omega}}{E}$ \\
\hline Vascular & $26(11 \%)$ & 0.002 & $6(21 \%)$ & $20(10 \%)$ & 结 \\
\hline Renal & $33(14 \%)$ & NS & $5(17 \%)$ & $28(14 \%)$ & $\frac{\bar{c}}{d}$ \\
\hline Paquetes globulares & $3 \pm 4$ & 0 & $8.6 \pm 5.5$ & $2.3 \pm 3.1$ & $\stackrel{\text { }}{\check{D}}$ \\
\hline Paquetes globulares $>6$ & $33(14 \%)$ & NS & $17(60 \%)$ & $16(8 \%)$ & 엉 \\
\hline \multicolumn{6}{|l|}{$\mathrm{UCl}$} \\
\hline UCI (días) & $2 \pm 5$ & 0 & $9.3 \pm 10$ & $1.16 \pm 3$ & 엉 \\
\hline $\mathrm{UCl}>7$ días & $26(11 \%)$ & 0 & $12(42 \%)$ & $14(7 \%)$ & $\frac{1}{2}$ \\
\hline
\end{tabular}


Tabla 2. Datos sociodemográficos y mortalidad asociada en pacientes con herida abdominal por proyectil de arma de fuego ( $n=230)$ (Continuación)

\begin{tabular}{|c|c|c|}
\hline & Genera & Anál \\
\hline & & Intestin \\
\hline Grado & Frecuencia & \\
\hline 1 & \multicolumn{2}{|c|}{0} \\
\hline 2 & \multicolumn{2}{|c|}{$11(15 \%)$} \\
\hline 3 & \multicolumn{2}{|c|}{$41(57 \%)$} \\
\hline 4 & \multicolumn{2}{|c|}{$17(24 \%)$} \\
\hline 5 & \multicolumn{2}{|c|}{$3(4 \%)$} \\
\hline \multicolumn{3}{|c|}{ Estómago } \\
\hline 1 & \multicolumn{2}{|c|}{$2(5 \%)$} \\
\hline 2 & \multicolumn{2}{|c|}{$26(70 \%)$} \\
\hline 3 & \multicolumn{2}{|c|}{$8(22 \%)$} \\
\hline$\geq 4$ & \multicolumn{2}{|c|}{$1(3 \%)$} \\
\hline \multicolumn{3}{|r|}{ Intestin } \\
\hline Localización & \multicolumn{2}{|c|}{ Total } \\
\hline Colon derecho & \multicolumn{2}{|c|}{34} \\
\hline Colon transverso & \multicolumn{2}{|c|}{25} \\
\hline Colon izquierdo & \multicolumn{2}{|c|}{22} \\
\hline Sigmoides & \multicolumn{2}{|c|}{12} \\
\hline Recto & \multicolumn{2}{|c|}{7} \\
\hline \multirow{2}{*}{\multicolumn{3}{|c|}{$\begin{array}{l}\text { EEATT: enteroentero anastomosis terminoterminal; NS: no significativo ( } p>0.05) \text {; RTS: Revise } \\
\text { Tabla 3. Variables de riesgo para estancia hospitalaria prolongada } \\
(\geq 14 \text { días })(n=230)\end{array}$}} \\
\hline & & \\
\hline & \multicolumn{2}{|r|}{ Hazard ratio } \\
\hline Frecuencia respiratoria $\leq 12$ & 0.023 & 73.442 \\
\hline Frecuencia cardiaca > 100 & 0.007 & 23.627 \\
\hline Saturación de oxígeno < 92\% & 0.071 & 8.863 \\
\hline Heridas $>1$ & 0.050 & 8.455 \\
\hline \multicolumn{3}{|l|}{ Cuadrantes abdominales } \\
\hline Superior derecho & 0.579 & 1.271 \\
\hline Superior izquierdo & 0.053 & 2.289 \\
\hline Inferior derecho & 0.081 & 2.332 \\
\hline Inferior izquierdo & 0.244 & 1.701 \\
\hline
\end{tabular}

tasas de complicaciones ${ }^{20}$. Muchas de estas medidas, especialmente la ISS, se basan en evaluaciones de lesiones en lesiones de múltiples regiones del cuerpo.
Se dispone de diversas medidas de gravedadj de la lesión para evaluar un TA penetrante ${ }^{18,20,21}$. $\$$ Las heridas de bala son características comunes de las lesiones traumáticas en muchas ciudades metropolitanas. Para los pacientes en países en vías de desarrollo pueden ser especialmente perjudiciāles debido a una falta general de atención prehospitâlaria y un difícil acceso a los servicios de salud, cömo ocurre en el país de donde proviene esta investigación ${ }^{22}$. Las HPPAF afectan de manera desproporcionada a pacientes adultos jóvenes durante sus años económicamente más productivos, lo que hace hecesario que se establezcan vías para mejorar tanto el manejo en urgencias como el quirúrgico y el posterior seguimiento ${ }^{1,6}$.

En la mayoría de los estudios realizados en el mundo desarrollado se ha reportado que el intestino delgădo es el órgano con más frecuencia lesionado en el TA 
penetrante. Adesanya, et al. ${ }^{19}$ y Feliciano, et al. ${ }^{5}$ informaron que el $52.4 \%$ y el $60 \%$ de sus pacientes tenían lesiones en el intestino delgado, respectivamente. En nuestro estudio, el intestino grueso estaba más comúnmente lesionado que el intestino delgado, aunque este último también era un órgano a menudo lesionado. Esto puede ser un reflejo de las tendencias divergentes de las heridas de bala; en particular, refleja el uso de armamento de alto calibre capaz de inducir daño a través de un segmento más grande de tejido interno ${ }^{21-23}$.

El cuadrante abdominal que más riesgo tuvo para una estancia hospitalaria prolongada fue el superior izquierdo, esto probablemente ligado a lesión en el bazo, que es el órgano más frecuentemente lesionado (30-40\%) en cuanto a TA se refiere. El dolor abdominal y el choque por hemorragia representan la presentación clínica más frecuente. La irritación peritoneal ocurre como complicación por daño de órganos huecos (intestino delgado, estómago, colon, epiplón). Por otro lado, el número de heridas representó una variable que incrementa 8.45 veces el riesgo para una estancia intrahospitalaria prolongada ${ }^{24}$, aumentando el riesgo de complicaciones como empiema asociado, infecciones urinarias y neumonías, deterioro de la calidad de vida y disminución de la optimización de recursos en esta área, además de un (ya documentado) aumento de la mortalidad ${ }^{25}$.

Hace falta correlacionar variables en diferentes tiempos quirúrgicos, además de incluir datos más específicos respecto a los distintos métodos terapéuticos utilizados en este estudio y datos obtenidos en la sala de urgencias, donde se lleva a cabo el primer contacto con los pacientes con TA por HPPAF. La reproducción de este estudio para futuras investigaciones beneficiará a la creación de estadística y estadificación del riesgo en países en vías de desarrollo y con preferencia a casos de violencia y a presentar este tipo de lesiones, lo que podría dificultar la atención médica temprana.

El TA por HPPAF es, en la actualidad, un tema que requiere atención multidisciplinaria, ya que es prioritario mejorar la calidad del servicio médico adecuándose a las situaciones poco favorables que prevalecen en los países en vías de desarrollo, además de mejorar la evolución terapéutica y contribuir a aumentar el estado de seguridad pública nacional.

\section{Conclusiones}

Los resultados demuestran la utilidad de la presencia de choque y el número de órganos abdominales dañados como predictores válidos de la gravedad de la lesión para un subgrupo crítico de TA por HPPAF en adultos y en adolescentes, además de evaluar los signos vitales y las características específicas de la lesión para la predicción de mortalidad por TA por HPËAF. Realizamos uno de los primeros reportes de HPPAF con un alto volumen de pacientes en México.

\section{Conflicto de intereses}

No existen conflictos de intereses entre los autores de este estudio.

\section{Financiamiento}

Los autores declaran que no se percibió apoyo ęconómico para la realización de esta investigación.

\section{Responsabilidades éticas}

Protección de personas y animales. Los autores declaran que para esta investigación no se han fealizado experimentos en seres humanos hi ${ }^{-}$en animales.

Confidencialidad de los datos. Los autores declaran que han seguido los protocolos de su centrê de trabajo sobre la publicación de datos de pacientes.

Derecho a la privacidad y consentimiento informado. Los autores han obtenido el consentimiènto informado de los pacientes y/o sujetos referidos en el artículo. Este documento obra en poder del autor-de correspondencia.

\section{Bibliografía}

1. Jadon D, Shaddick G, Jobling A, Ramanan AV, Sengupta R. Clinical outcomes and progression to orthopedic surgery in juvenile versus adult-onset ankylosing spondylitis. Arthritis Care Res (Hoboken). 2015;67:651-7.

2. Lozano R, Naghavi M, Foreman K, Lim S, Shibuya K, Aboyans V,et al. Global and regional mortality from 235 causes of death for 20 age groups in 1990 and 2010: a systematic analysis for the Global Burden of Disease Study 2010. Lancet. 2012;380:2095-128.

3. Dawidson I, Miller E, Litwin MS. Gunshot wounds of the abdomen. A review of 277 cases. Arch Surg. 1976;111:862.

4. Demetriades D, Charalambides D, Lakhoo M, Pantanowitz D. Gü $\overline{\bar{m}}$ shot wound of the abdomen: role of selective conservative management. $\mathrm{Br}$ J Surg. 1991;78:220.

5. Feliciano DV, Burch JM, Spjut-Patrinely V, Mattox KL, Jordan GL Jr. Abdominal gunshot wounds. An urban trauma center's experience with 300 consecutive patients. Ann Surg. 1988;208:362.

6. World Health Organization. World report on violence and health. Geneva: WHO; 2002.

7. Cocanour CS, Fischer RP, Ursic CM. Are scene flights for penetrating trauma justified? J Trauma. 1997;43:83. Discussion 6e8.

8. Haygood FD, Polk HC Jr. Gunshot wounds of the colon. A review of 100 consecutive patients, with emphasis on complications and their cayses. Am J Surg. 1976;131:213.

9. Payne JE, Berne TV, Kaufman RL, Dubrowskij R. Outcome of treatment of 686 gunshot wounds of the trunk at Los Angeles County-USC Medical Center: implications for the community. J Trauma. 1993;34:276. 
10. Annis P, Lawrence BD, Spiker WR, Zhang Y, Chen W, Daubs MD, et al. Predictive factors for acute proximal junctional failure after adult deformity surgery with upper instrumented vertebrae in the thoracolumbar spine. Evid Based Spine-Care J 2014;5:160-2.

11. Espinosa A, Stenseth R, Videm V, Pleym H. Comparison of three point-ofcare testing devices to detect hemostatic changes in adult elective cardiac surgery: a prospective observational study. BMC Anesthesiol. 2014;14:80

12. Parra-Romero G, Contreras-Cantero G, Orozco-Guibaldo D, Domínguez-Estrada A, Mercado-Martín del Campo JJ, Bravo-Cuéllar L. Traumatismo abdominal: experiencia de 4961 casos en el occidente de Mexico. Cir Cir. 2019;87:183-9.

13. De Giorgi S, De Giorgi G, Borracci C, Tafuri S, Piazzolla A, Moretti B. Adult scoliosis: age-related deformity and surgery. Eur Spine J. 2014;23(Suppl 6):597.

14. Sacco WJ, Champion HR, Gainer PS, Morelli SA, Fallen S, Lawnick MA The trauma score as applied to penetrating trauma. Ann Emerg Med. 1984;13:415.

15. Champion HR, Sacco WJ, Carnazzo AJ, Copes W, Fouty WJ. Trauma score. Crit Care Med. 1981;9:672.

16. Champion HR, Copes WS, Sacco WJ, Frey CF, Holcroft JW, Hoyt DB, et al. Improved predictions from a severity characterization of trauma (ASCOT) over Trauma and Injury Severity Score (TRISS): results of an independent evaluation. J Trauma. 1996;40:42-8. Discussion 8e9.
17. Copes WS, Champion HR, Sacco WJ, Lawnick MM, Keast SL, Bain LW. The injury severity score revisited. J Trauma. 1988;28:69.

18. Moore EE, Dunn EL, Moore JB, Thompson JS. Penetrating abdominal trauma index. J Trauma. 1981;21:439.

19. Adesanya AA, da Rocha-Afodu JT, Ekanem EE, Afolabi IR. Factors affecting mortality and morbidity in patients with abdominal gunshot wounds. Injury. 2000;31:397.

20. Croce MA, Fabian TC, Stewart RM, Pritchard FE, Minard G, KudsK KA. Correlation of abdominal trauma index and injury severity score with abdominal septic complications in penetrating and blunt trauma. STrauma. 1992; 32:380. Discussion 7e8.

21. Gorea RK. Medical and social aspects of proliferation of small arms and light weapons in South Asia. J Indian Acad Forensic Med 2004;26(2).

22. Memon AA, Bhutto AA, Shaikh GS, Jhokio A, Soomro Q. Pattern of firearm injury and outcome. JLUMHS. 2009:8:143.

23. Khetran AK, Rehman S, Khan Z, Baloch MR. Incidence of deaths due to gunshot injuries at District Barkhan, Balochistan. JLUMHS. 2012;71:90.

24. Concha Torre A, Rey Galán C, Rodríguez Suárez J. Serie monográfica. Manejo inicial del politraumatismo pediátrico (IV). Traumatismo abdominal. Bol Pediatr. 2009;49:58-68.

25. López Pardo P, Socorro García A, Baztán Cortés JJ. Influencia de đà duración de la estancia hospitalaria sobre la mortalidad tras el alta en pacientes mayores con patología médica aguda. Gac Sanit. 2016;30:375-8. 